\title{
Carbon Stars With Oxygen-Rich Circumstellar Chemistry
}

\author{
M.S. Vardya \\ Tata Institute of Fundamental Research
}

\begin{abstract}
Ten new carbon stars with oxygen-rich circumstellar shells have been discovered using IRAS LRS spectral class. The origin of these objects have been briefly discussed. It is likely that they may be a consequence of release of oxygen due to carbon condensation, though other scenarios cannot be ruled out.
\end{abstract}

\section{Introduction}

IRAS LRS spectra show $9.7 \mu \mathrm{m}$ silicate emission in a large number of stars. The existence of this feature indicates oxygen-rich circumstellar (CS) chemistry. The presence of this feature is denoted in the LRS spectral classification (Olnon and Raimond 1986) by $2 \mathrm{n}$ and $6 n$, where $n$ is a measure of the strength of the feature, and is given in the IRAS point source catalogue as well. Presence of this feature is expected in M stars but not in carbon stars. Therefore, it was a complete surprise when Little-Marenin (1986), Little-Marenin and Wilton (1986), and Willems and de Jong (1986) found ten carbon stars, including a Mira, with silicate emission. This discovery leads to several interesting possibilities about the existence and evolution of such objects.

The Sample

In order to increase the sample of such carbon stars, we have used the IRAS LRS spectral class along with the carbon star (Stephenson 1973) and variable star (Kholopov 1985) catalogues. In this way, we have discovered nine additional stars, all having LRS spectral class 2n. RT Oph, a Mira variable, can also be added to this list if its spectral classification (Kholopov 1985) of $M 7 e(C)$ is confirmed. Note that the strength of the silicate emission, as denoted by $n$, is weak in a few cases, and may have resulted from noise in the spectra.

\section{Results}

The table gives the complete list of these carbon stars with oxygen-rich circumstellar shells discovered so far. The first ten 
Carbon Stars With Oxygen Rich Circumstellar Shell
R.A.
(1950)
$\begin{array}{ll}\text { Decl. } & \text { ocs Name } \\ \text { (1950) } & \begin{array}{l}\text { No. } \\ \end{array}\end{array}$
Sp.
class

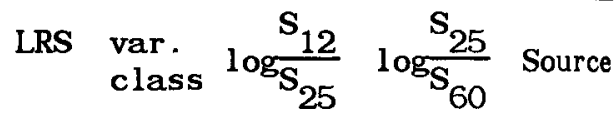

1. $001714.9+442553$

11 VX And

C4,5J $24 \quad$ SRa

$0.534 \quad 0.586$

$\mathrm{V}$

2. $011321.1+253019$

$63 \mathrm{Z}$ Psc

(N7)

C7,2

$22 \mathrm{SRb}$

0.473

0.541

(NO)

3. $044822.5+282634$

254 TT Tau

$\mathrm{C} 4,2-7,422 \quad \mathrm{SRb}$

0.492

0.218

(N3)

4. $071755.2+250540$

716 BM Gem

C5,4J

$29 \quad \mathrm{SRb}$

0.252

0.845

$(\mathrm{Nb})$

5. $080017.0+380330$

1003 --

--

$27 \quad-$

$-0.042$

$0.568 \mathrm{~L}, W$

6. $085348.8+200230$

1344 T Cnc

C3,8-5,5 $23 \quad$ SRb

0.570

0.654

V

7. $085745.6-603554$

-- MC79-11 (R6-N6)

8. $100906.5-704925$

1633 --

$\mathrm{N}, \mathrm{Mb}$

$65-$

0.016

0.702

WD

9. $\quad 133421.7-561321$

2106 RV Cen

N3e

$22 \quad-$

0.368

0.821

WD

10. $134417.7-610930$

2123 --

C: :

$25 \quad M$

0.460

0.583

LW

11. $175411.9+111031$

-- $\quad$ RT Oph

$\operatorname{Mre}(\mathrm{C}) \quad 22 \quad \mathrm{M}$

0.135

0.710 WD

12. $180037.1-321310$

-- FJF270

--

22 --

0.339

$0.787 \quad \mathrm{~V}$

13. $185614.9+141738$

2684 UV Aql

C5,4-5 $22 \quad$ SRa

$-0.051$

0.409 WD

14. $191355.3+541206$

-- NC\#83

(N4)

15. $192322.7+762746$

2738 UX Dra

C7, 3

28 --

0.536

0.456

$\mathrm{V}$

16. $192454.7+232948$

2733

C5, 4

(N)

17. $194140.8+342209$

$2783 \quad-$

C

0.278

0.938 WD

18. $203504.0+595456$

2919 v778 Cyg

C4,5J $29 \quad \mathrm{Lb}$ (N)

19. $231737.3+465802 \quad 3184$ EU And

C4, 4

29 SR

0.533

0.618

$\mathrm{V}$

(R)

20. $235842.4+6004393214$ WZ Cas

$\begin{array}{lll}\text { C9,2JLi } & 23 & \mathrm{SRb}\end{array}$

0.509

0.550

$\mathrm{V}$ 
Notes on the table:

1. $\mathrm{P}=369 \mathrm{~d} ; \dot{\mathrm{M}}>1.6 \times 10^{-7} \mathrm{M}_{\odot} / \mathrm{yr}$ (Olof sson et al .1987$) ; \mathrm{T}_{\mathrm{NIR}}=$ $2400 \mathrm{~K}$ (Willems 1987)

2. $P=144 \mathrm{~d}, \dot{\mathrm{M}}=0.4 \times 10^{-7} \mathrm{M}_{\odot} / \mathrm{yr} ; \mathrm{T}_{\mathrm{NIR}}=2800 \mathrm{~K}$

3. $P=167 \mathrm{~d}(\mathrm{P}=364 \mathrm{~d} ?) ; \mathrm{T}_{\mathrm{NIR}}=2550 \mathrm{~K}$

4. Periodic variation during some intervals, and shortly thereafter, not variable

6. $P=482 \mathrm{~d} ; \mathrm{f}=0.35 ; \mathrm{T}_{\mathrm{NIR}}=2250 \mathrm{~K}$

7. $\left(\mathrm{OH}, \mathrm{H}_{2} \mathrm{O}\right)$-maser

8. S Star?

9. $\quad P=446 \mathrm{~d}, \mathrm{f}=0.56$

10. $\mathrm{H}_{2} \mathrm{O}$ maser

11. $P=426 \mathrm{~d}$ but variable; $f=0.36 ;\left(\mathrm{OH}, \mathrm{H}_{2} \mathrm{O}\right)$-maser; needs to be confirmed as a carbon star

12. Wrongly put as FJF 272

13. $P=386 \mathrm{~d} ; \mathrm{T}_{\mathrm{NIR}}=2350 \mathrm{~K}$

15. $P=168 \mathrm{~d}, f=0.5 ; \mathrm{M}=1.8 \times 10^{-7} \mathrm{M}_{\odot} / \mathrm{yr} ;$ light curve may correspond to type E; SB; $\mathrm{P} \sim 340 \mathrm{~d}$

16. $\mathrm{T}_{\mathrm{NIR}}=2200 \mathrm{~K}$

17. $\mathrm{N}_{\mathrm{NIR}}=1200 \mathrm{~K}$

18. $\mathrm{H}_{2} \mathrm{O}$ maser

19. $\mathrm{H}_{2} \mathrm{O}$ maser

20. $\mathrm{P}=186 \mathrm{~d} ; \mathrm{T}_{\mathrm{NIR}}=2650 \mathrm{~K} ; \mathrm{VB} \mathrm{A} ;$ light variations are sometimes taking place with double period; hydrogen emission not always detected; $\mathrm{H}_{2}$ IR line detected 
columns give serial number, right ascension and declination (epoch 1950) Stephenson's (1973) cool carbon star catalogue number. star name, spectral classification, IRAS LRS spectral class, type of variability, $\log S_{12} / S_{25}$, and $\log S_{25} / S_{60}$. Here $S_{\lambda}$ is the flux density at

wavelength $\lambda$ in microns from the IRAS point source catalogue. The last column gives the source of discovery - L for Little-Marenin (1986), LW for Little-Marenin and Wilton (1986), V for this paper and WD for Willems and de Jong (1986). Notes at the end of the table give additional information on some of these stars. The spectral classification, especially the $C$ subclass, is not available for about half the stars.

The figure gives a plot of $\log \left(\mathbf{S}_{12} / \mathrm{S}_{25}\right)$ vs $\log \left(\mathbf{S}_{25} / \mathrm{S}_{60}\right)$. Solid line is the blackbody curve. The number at the top of the star gives the LRS spectral class. The figure shows that most of the sources with weak or moderate strength of silicate emission lie near or below the blackbody line, as is true for normal carbon stars (cf. Willems 1987). However, all sources with spectral class $2 n$, with $n>7$ are above the blackbody line, with smaller values of $\log \left(S_{12} / S_{26}\right)$ and larger values of $\log \left(\mathrm{S}_{25} / \mathrm{S}_{60}\right)$. A line above the blackbody line but slightly inclined to it demarcates the two regions. Note that class $6 \mathrm{n}$ may behave slightly differently. The dispersion of $\log \left(\mathrm{S}_{12} / \mathrm{S}_{25}\right)$ for weak

silicate sources is small, barring a few exceptions, but is large for strong sources. In this sense also, weak stlicate sources mimic normal carbon stars. The silicate emission strength appears to increase with increase in $\log \left(S_{12} / S_{25}\right)$ in the strong silicate sources.

\section{Discussion}

In an attempt to understand the causes of carbon stars showing oxygen-rich circumstellar dust, several possibilities have been advanced by Little-Marenin (1986) and by Willems and de Jong (1986).

1. An unusual chemical equilibrium exists in the $C S$ matter, such that an oxygen-rich environment is produced out of carbon-rich material due to interaction of graphite and $\mathrm{SiC}$ grain formation with $\infty$.

2. Due to rapid $M \rightarrow S \rightarrow C$ star evolution, the $s$ tar has become a $C$ star since it (as an $M$ star) ejected the oxygen-rich grains.

3. The system is a binary in which the $\mathrm{C}$ star is brighter, but the $M$ star is the mass ejector.

4. This has been rapid, straight $M \rightarrow C$ star evolution, with oxygen-rich grains produced at the end of the M-star phase, which has now been ended by the conversion to a carbon star, as in 2 above. 


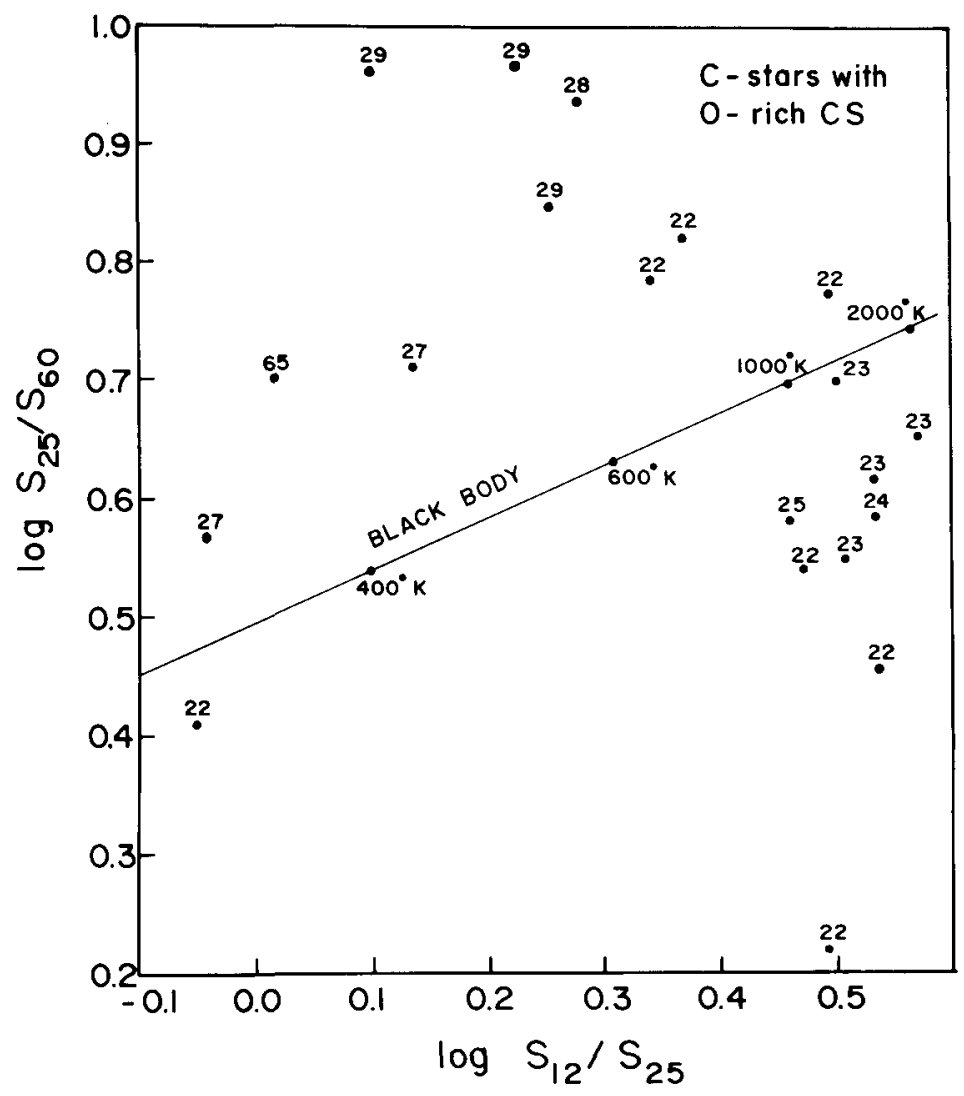

A color-color plot -- $\log \left(\mathrm{S}_{25} / \mathrm{S}_{60}\right)$ vs $\log \left(\mathrm{S}_{12} / \mathrm{S}_{25}\right)$-- for carbon stars with oxygen-rich circumstellar shells. The number above each star gives the IRAS LRS spectral class. For comparison the solid line denotes a blackbody curve with various relevant temperatures marked. 
Little-Marenin (1986) has discussed the first three possibilities but prefers the binary system hypothesis. The observation of water maser emission towards EU And (star no. 19) and the differences in velocity between photospheric absorption lines and the $\mathrm{H}_{2} \mathrm{O}$ maser line have led Benson and Little-Marenin (1987) to propose it as the first radio spectroscopic binary, with the $C$ star dominating the optical spectrum and the M star the far infrared; however, the binary nature is yet to be confirmed by observing periodic change in the radial velocity and the fact that the other component is an $M$ star. Note that only two or three are known binary systems in our sample.

Willems and de Jong (1986) have also discussed the binary possibility but ruled it out; they prefer direct evolution from $M$ to $C$ star without passing through the intermediate S-star phase, especially for J-type carbon stars with ${ }^{13} \mathrm{C}$ overabundance. In the table only four stars are known to be J-type, two with strong silicate emission and two with silicate emission of moderate strength. The Mira variable RT Oph was classified earlier as M7e (Kukarkin et al. 1969) but is now classified as M7e (C) (Kholopov 1985); if this is indeed a carbon star, it may be pointing to the scenario suggested by Willem and de Jong (1986).

The above two possibilities, though not ruled out, may not be the answer for all such objects. But the solution via chemical equilibrium (no. 1), if it works, will have wide applicability. Tarafdar (1987) has carried out molecular equilibrium calculations with condensation for carbon-rich elemental composition; these show that condensation can increase the abundance of certain oxygen bearing compounds by several orders of magnitude, giving the appearance of oxygen-rich chemistry. Such calculations, but based on realistic conditions pertaining to circumstellar shells, may lead to a true picture. Such calculations may also hold the mystery of the presence of HCN in the oxygen rich circumstellar shells (cf. Deguchi and Goldsmith 1985).

\section{Conclusion}

Search for more carbon stars with oxygen-rich circumstellar shells should be made, and the known ones should be subjected to more detailed observations, besides theoretical calculations for condensation in a carbon-rich circumstellar conditions be undertaken. Then we may know the true nature of these objects.

\section{References}

Benson, P.J. and Little-Marenin, I.R. 1987, Astrophys. J. (Lettr.). 316, L37.

Deguchi, S., and Goldsmi th, P.F. 1985, Nature, 317, 336.

Kholopov, P.N. 1985, General Catalogue of Variable Stars, 4th ed., (Moscow. Nauka, Publ. House), Vols. I-III. 
Kukarkin, B.V. et al., General Catalogue of Variable Stars, 3rd ed. and supplements (Moscow: Astronomical Council of the Academy of Sciences of the USSR).

Little-Marenin, I.R. 1986, Astrophys. J. (Lettr.) 307, L15.

Little-Marenin, I.R., and Wilton, C. 1986, in Cool Stars, Stellar Systems, and the Sun, ed. M. Zeilik and D.M. Gibson (Berlin: Springer Verlag), p. 420.

Olofsson, H., Eriksson, K., and Gustafsson, B. 1987, Astron. Astrophys. $183,413$.

Olnon, F.M. and Raimond, E. 1986, Astron. Astrophys. Suppl. 65, 607.

Stephenson, C.B. 1973, A General Catalogue of Cool Carbon Stars, Publ. Warner and Swasey Obs., Vol. 1, No. 4.

Tarafdar, S.P. 1987, Astrochemistry, eds. M.S. Vardya and S.P. Tarafdar, (Dordrecht: D. Reidel Publ. Co.), p. 589.

Willems, F.J. 1987, Infrared Studies of Asymptotic Giant Branch Stars, $\mathrm{Ph} . \mathrm{D}$. Thesis, University of Amsterdam.

Willems, F.J. and de Jong. T. 1986, Astrophys. J. (Lettr.) 309, L39. 